\title{
Primer la Llar: aplicación del modelo Housing First en la ciudad de Barcelona. Primeras reflexiones
}

\section{Carme Fortea Busquets}

Departamento de Atención a Personas Vulnerables, Ayuntamiento de Barcelona <cfortea@bcn.cat>

\section{Lourdes Herruz Pamies}

Programa Primer la Llar, Departamento de Atención a Personas Vulnerables, Ayuntamiento de Barcelona

<lherruz@bcn.cat>

Bartzelonako Udalak 2015eko ekainean abian jarri zuen Primer la Llar (Lehenik Etxebizitza) egitasmoa, Housing First ereduaren proba pilotu bat. Eskuartze sozialerako metodologia honek oinarri hartzen du kalean lo egiten duten etxegabeko pertsonentzako etxebizitza egonkor baterako zuzeneko sarbidea eta erabakiak hartzeko mugagabeko eperik ez duen lagun-egitea. Lehenik Ipar Amerikan eta geroago Europako herrialde batzuetan eredu hau arrakastatsua gertatu denez, gehienbat, programa honen helburua izan da Bartzelonako hirira egokitzeko informazioa bildu eta hiri honetan gaur egun indarrean dagoen etxegabeko pertsonentzako arreta-eredura egokitzea. Artikulu honetan deskribatzen da programa garatzen ari den testuingurua eta abian jartzeko definitu izan diren parametroak zeintzuk diren, eta horren ostean zenbait ohar aipatzen dira programak izandako funtzionamenduaren inguruan.

\section{GAKO-HITZAK:}

Housing First, etxegabeak, etxebizitza, pertsona ardatz duen eskuartze soziala, autonomia, ahalduntzea.
En junio de 2015, el Ayuntamiento de Barcelona puso marcha Primer la Llar (Primero el Hogar), una prueba piloto del modelo Housing First. Esta metodología de intervención social se basa en el acceso directo a la vivienda estable de personas sin hogar que duermen en la calle, así como en un acompañamiento a sus decisiones, sin límite temporal. Dado que este modelo ha sido ensayado con éxito, primero en Norteamérica y más recientemente en varios países europeos, el objetivo del programa era más bien obtener información sobre cómo adaptarlo a la ciudad y cómo encajarlo con el modelo en escala vigente hoy día en la atención a las personas sin hogar en Barcelona. El presente artículo describe el contexto en el que se desarrolla el programa, así como los parámetros que han definido su puesta en marcha, para después hacer algunas consideraciones sobre su funcionamiento.

\section{Palabras clave:}

Housing First, sinhogarismo, vivienda, intervención social centrada en la persona, autonomía, empoderamiento. 


\section{Introducción}

Barcelona dispone de servicios y recursos dirigidos a personas sin hogar consolidados y considerados de gran relevancia en el ámbito europeo. Servicios y recursos basados en el modelo en escala o de transición, articulado de manera conjunta y compartida por las distintas entidades de la ciudad, que cuentan con larga trayectoria en intervención con personas sin hogar. A partir de 2010, empezamos a recibir información sobre una nueva manera de enfocar la intervención con personas sin hogar y, muy especialmente, con aquellas en situación más cronificada en calle: el modelo Housing First (Tsemberis y Eisenberg, 2000).

La sociedad ha ido cambiando, el perfil de las personas sin hogar, también, y la forma de atención a las personas que se encuentran en esta situación se ha ido adaptando hacia un enfoque más próximo, más digno, más participativo y que pone a la persona en el centro de la intervención. Las personas sin hogar continúan siendo el sector más frágil de la exclusión social, pero ya no se las culpabiliza de su situación, sino que se empieza a orientar la atención desde la perspectiva del acceso a los servicios, considerando a los usuarios sujetos de pleno derecho.

En este marco del pleno derecho y de cambios en la intervención social, se desarrolla el planteamiento del programa Pathways to Housing, planteado en Nueva York (EE.UU.) por Sam Tsemberis en los años noventa (Tsemberis, 2010a, 2010b; Tsemberis y Eisenberg, 2000), aplicado después en Canadá y recientemente en Europa.

Las valoraciones realizadas en Europa sobre el funcionamiento del modelo Housing First, así como la evidencia de las dificultades inherentes a la atención a las personas en situación de sinhogarismo cronificada y con enfermedad mental o drogodependencia, han abierto las puertas a que en nuestro país se empiecen a plantear algunas prácticas en esta línea.

El Ayuntamiento de Barcelona inició su propio programa municipal de Housing First, con el nombre de Primer la Llar, en junio de 2015, siendo el primer ayuntamiento en desarrollarlo de forma directa. El municipio de Barcelona plantea su puesta en marcha manteniendo el resto de servicios de atención a personas sin hogar, con el objetivo de analizar los resultados de esta prueba piloto, adaptada a las características de la ciudad, y el ajuste del nuevo modelo en convivencia con el modelo actual en escala o de transición. En junio de 2014, el Ayuntamiento de Barcelona firmó un convenio con RAIS Fundación, que, a través de su programa Hábitat, implementaba el modelo Housing First en tres ciudades: Madrid, Málaga y Barcelona.

El objetivo del presente artículo es dar a conocer cómo y en qué contexto el Ayuntamiento de
Barcelona ha puesto en marcha el programa Primer la Llar, y cuáles son las primeras valoraciones y reflexiones tanto de los profesionales como de las personas participantes. La finalización de la prueba piloto y la obtención de resultados y análisis globales de la evaluación de impacto y de calidad están previstas para finales de 2018.

\section{Sinhogarismo y Housing First: marco legal}

En la ciudad de Barcelona, la atención a personas sin hogar tiene una larga trayectoria, que se remonta a principios del siglo pasado (Fortea, 2005). En un principio, el tipo de atención que recibían las personas sin hogar estaba vinculada al asistencialismo. Beneficencia y mendicidad iban de la mano, y así se mantuvo durante mucho tiempo. En 1979, en Barcelona existían dos albergues, Valldonzella y Sant Joan de Déu, que permitían atender las demandas de atención de este colectivo, amparadas en la Ley de Vagos y Maleantes.

A partir de 1985, con la primera Ley de Servicios Sociales catalana, la atención da un giro importante, creándose nuevos recursos con mucha más dignidad. A principios de los noventa, se pusieron en marcha los primeros equipos de calle, centros de día, centros para la acogida invernal, la Operación Frío, el centro Can Planas, y nuevos albergues o centros de alojamiento. Posteriormente, se incorporaron las viviendas de inclusión y, por último, en 2015 comenzó a implementarse el nuevo modelo Housing First.

Gracias al Acuerdo Ciudadano para la Barcelona Inclusiva y a la creación del Programa Municipal de Atención a las Personas Sin Techo, en 2005. el Ayuntamiento ha ido ampliando los recursos y servicios y consolidando la colaboración con las entidades sociales a través de la Xarxa d'Atenció a Persones Sense Llar (XAPSLL) ${ }^{1}$. Recientemente, en el marco del Programa de Actuación Municipal 20162019, el Ayuntamiento ha elaborado el Pla de Lluita contra el Sensellarisme de Barcelona 2016-2020², fruto de un amplio proceso participativo en el que han intervenido también las personas que están en situación de sinhogarismo, y en este documento ya no se habla de un colectivo específico, sino de una situación de sinhogarismo que vulnera el derecho básico al acceso a la vivienda. La intervención se debe orientar al acceso a la vivienda y al alojamiento, y es en este marco en el que los programas Housing First juegan un importante papel en la lucha contra el sinhogarismo.

La Unión Europea, en la Estrategia 2020, se compromete a reducir en veinte millones el número de personas en situación de pobreza en los Estados

\footnotetext{
${ }^{1}$ Red de Atención a las Personas sin Hogar.

2 Plan de Lucha contra el Sinhogarismo de Barcelona 2016-2020.
} 
miembros. Por su parte, la Comisión Europea, en su conjunto de medidas sobre inversión social (Social Investment Package), coloca la provisión de vivienda en el centro de la intervención para combatir el sinhogarismo de los Estados miembros. Finalmente el Parlamento Europeo aprobó dos resoluciones instando a la Comisión a elaborar una estrategia europea y animando a los Estados miembros a diseñar las suyas propias.

El Estado Español aprobó en 2015 la Estrategia Nacional Integral para Personas Sin Hogar 20152020, con el objetivo de reducir el sinhogarismo a través de la prevención, el alojamiento y de un enfoque centrado en las personas; también a través de la implementación de proyectos Housing First. El Gobierno de la Generalitat, a 20 de diciembre de 2016, ha aprobado un Acuerdo de Gobierno que impulsa el diseño de la Estrategia Integral para el Abordaje del Sinhogarismo en Cataluña y también prevé la realización de pilotos basados en la metodología Housing First.

\section{El Programa Municipal de Atención a Personas sin Hogar y el modelo Housing First}

El Programa Municipal de Atención a las Personas Sin Hogar, articulado como tal en 2005, está basado en un modelo en escala y, según la clasificación ETHOS $^{3}$, se centra en la atención a las personas que se encuentran viviendo en la calle, en centros de alojamiento específicos o en vivienda inadecuada, concretamente en caravanas. Los servicios que configuran el programa incluyen equipos de calle, equipos de atención social, centros de alojamiento, centros de día, servicios de higiene y comedores sociales, todos ellos de gestión externalizada. Existen diferentes puertas de entrada al programa, y las personas pueden acceder a algunos de los servicios directamente, sin el acompañamiento de un profesional. Se trabaja a partir de un expediente único, lo que permite hacer un seguimiento de la trayectoria de las personas atendidas. El programa es de ámbito municipal, y se establecen coordinaciones con cada uno de los territorios, con los servicios sociales y con el tejido asociativo, a través del trabajo con XAPSLL y con la Xarxa Habitatges Inclusió (XHI) ${ }^{4}$.

Desde 2008, la XAPSLL recoge sistemáticamente y analiza los datos de la situación de sinhogarismo de la ciudad a través de recuentos e informes periódicos, que juntamente con los informes de los equipos municipales de calle, confirman que el número de personas que duermen en la calle ha ido aumentando: concretamente, en 2008 había 608, y en 2016 se contabilizan 941 personas, lo que supone un incremento del $37 \%$. En el mismo periodo, las personas que dormían en recursos especializados pasaron de 1.190 a 1.907, un aumento del $60 \%$. Es evidente que se ha realizado un gran esfuerzo para contener el crecimiento de la exclusión residencial.

Los servicios se han ido diversificando y dignificando, potenciando el papel de la persona como el centro de la propia inserción. Se han dignificado las infraestructuras, priorizando espacios individuales y adaptados, y fomentando la participación de las personas.

Tal y como se recoge en el Pla de Lluita contra el Sensellarisme de Barcelona 2016-2020, tanto el Ayuntamiento de Barcelona como la XAPSLL llevan años intentando superar la perspectiva clásica de atención a las personas sin hogar, poniendo el foco de la atención en la falta de vivienda. Para rehacer una vida tras haber experimentado una situación de calle, es necesaria una estabilidad económica y habitacional que permita la estabilidad emocional necesaria para iniciar un proceso de recuperación. Las políticas Housing Led orientan la intervención de los servicios sociales a la reconstrucción del hogar en un sentido amplio, buscando el empoderamiento de la persona a través de la reconstrucción de vínculos afectivos y sociales que sólo pueden producirse si se dispone de los espacios para ello.

Así pues, el Ayuntamiento de Barcelona argumenta la puesta en marcha del modelo Housing First a partir de los siguientes elementos:

- El Ayuntamiento de Barcelona es miembro de la asociación Eurocities 5 , y participa en las reuniones periódicas del grupo de trabajo de sinhogarismo. En este grupo, a partir de 2010 se empezó a recibir información de la existencia del modelo Housing First y de la buena valoración hecha por las ciudades que lo habían implementado. Se hicieron visitas a Francia, Portugal y los Países Bajos. También se promovieron sesiones de trabajo en Barcelona con expertos en el modelo: Tania Tull (California, Path Beyond Shelter), Dennis Culhane (Universidad de Pensilvania), José Ornelas (Lisboa), Equipo Chez Soi d'Abord (París), Roch Hurtubise (Quebec, Chez Soi), Sam Tsemberis (Nueva York, Pathways to Housing), Y Foundation (Finlandia).

- La ciudad de Barcelona dispone de un modelo de intervención municipal en escala para personas sin hogar consolidado y consensuado en la XAPSLL. Sin embargo, algunas personas sin hogar en situación crónica no se vinculan a los recursos existentes, utilizándolos de manera puntual, con una recurrencia calculada de en torno al $34 \%$.

- En la XAPSLL, hubo consenso para la puesta en marcha de la experiencia en la ciudad y se realizó una importante labor de incidencia para su implementación, en especial por parte de aquellas entidades vinculadas a la Federación

\footnotetext{
3 Tipología europea de sinhogarismo de Feantsa (2005).

${ }_{4}^{4}$ Red de Viviendas de Inclusión.
}

${ }^{5}$ Eurocities es un red de grandes ciudades europeas creada en 1989 para compartir experiencias y elaborar respuestas a escala local. 
Europea de Asociaciones Nacionales que Trabajan con Personas sin Hogar (Feantsa), organismo que ha promovido la implementación del modelo en Europa.

El Ayuntamiento de Barcelona diseña el proyecto de Primer la Llar en 2014, basado en el modelo Pathways to Housing (también llamado 'modelo puro'), aunque tal y como describe Nicolas Pleace (2012) se pueden distinguir tres enfoques principales del modelo:

- Pathways (PHF): orientado a personas sin hogar en situación crónica y con vivienda dispersa.

- Communal Housing (CHF): orientado a personas sin hogar en situación crónica y con vivienda en un mismo edificio y con servicios comunes.

- Housing First Led (HFL): orientado a otros colectivos sin hogar con menores necesidades de soporte).

En el primer trimestre de $\mathbf{2 0 1 5}$, se realiza un concurso público para la adjudicación del programa, planteado para 50 viviendas, y seguidamente se procede a la propuesta y selección de los candidatos. El 1 de junio de 2015 se inicia la implementación del programa.

\section{Primer la Llar Barcelona: programa municipal Housing First}

\subsection{Definición}

\subsubsection{Finalidad}

El programa Primer la Llar de Barcelona ofrece una vivienda permanente con apoyo y seguimiento socioeducativo a personas sin hogar y en situación de exclusión social severa, mediante la aplicación del modelo de intervención Housing First. El programa se dirige a personas con una situación de sinhogarismo cronificada, con larga trayectoria de vida en la calle o puntualmente en recursos residenciales, que no han establecido vinculación y presentan problemas graves de salud mental o adicciones.

La finalidad es iniciar un proceso de recuperación y mejora de la calidad de vida de la persona para que pueda permanecer en la vivienda sin más apoyo que la red normalizada de servicios, para lograr la autonomía personal y económica, la recuperación de habilidades personales de cuidado propio, el restablecimiento de relaciones sociales y familiares, y una mejora de la vinculación con el entorno.

Primer la Llar sigue los principios que fundamentan el modelo Housing First, basado en la separación del proceso de atención del de acceso y mantenimiento de la vivienda:

- Respecto a la vivienda, los aspectos fundamentales del modelo son:

- El piso es la vivienda permanente de la persona.
- Los pisos son independientes e individuales, y están dispersos por el municipio: se busca la desinstitucionalización y la normalización de las condiciones de vida.

- Respecto a la atención, cabe destacar que:

- La persona es el eje central de la atención: decide cuándo y qué quiere hacer.

- El profesional acompaña las decisiones, orienta, da soporte y acompañamiento, y facilita el empoderamiento.

- Individualización y personalización del soporte: respeto y empatía. No hay límite de tiempo.

- Orientación a la recuperación y reducción de daños.

- El equipo de soporte se desplaza a los pisos. Es multidisciplinario (social y sanitario) e incluye la figura del par (peer).

\subsubsection{Personas destinatarias}

El programa se dirige a personas sin hogar en situación crónica que viven en la calle o que no acaban de vincularse con los recursos existentes, utilizándolos sólo de manera puntual:

1. Personas solas mayores de edad (excepcionalmente, parejas sentimentales o de amigos/as en relación de soporte positiva).

2. Larga trayectoria de vida en la calle (mínimo un año pernoctando de manera continuada en la calle o en centros de alojamiento sin vincularse, utilizándolos de manera puntual o intermitente en el último año; sin mejora en el proceso ni aceptación de las normas, mero uso instrumental de cobertura de las necesidades básicas).

3. Necesidades sociales concurrentes a la falta de vivienda: enfermedad mental o adicciones.

4. Autonomía funcional para la vida diaria.

5. Disponer de ingresos económicos regulares o posibilidad de conseguirlos.

\subsubsection{Condiciones acceso}

Los candidatos a participar en el programa fueron propuestos por los servicios y recursos municipales de atención a personas sin hogar, así como por todas las entidades que forman parte de la XAPSLL. Los compromisos de los participantes fueron:

- Aceptar soporte profesional (contacto mínimo semanal).

- Respetar la convivencia vecinal y de la comunidad.

- Contribuir al coste de la vivienda (si dispone de ingresos).

- Aceptar el seguimiento y evaluación que se determine. 


\subsection{Gestión y recursos}

\subsubsection{Tipo de gestión}

Se realizó un concurso abierto para poner en marcha 50 viviendas en modelo Housing First. Se planteó la gestión en dos lotes de 25 viviendas, cada uno con las funciones de contratar y gestionar el equipo social, ocuparse de la búsqueda de viviendas, del alquiler de éstas, de su equipamiento (mobiliario, adecuación) y consumos, así como de gestionar ayudas económicas y el pago de los participantes en concepto de alquiler.

El 1 de junio de 2015 se puso en marcha el programa piloto municipal Primer la Llar, con una implementación gradual hasta junio de 2016, fecha en que estaban en funcionamiento las 50 viviendas previstas. El plazo de ejecución de contrato es el 31 de mayo de 2018, prorrogable hasta el 31 de diciembre de ese año. Cada uno de los dos lotes del programa está adjudicado a una entidad: el primero, a Sant Joan de Déu Serveis Socials; y el segundo, a la UTE formada por Suara, Sant Pere Claver y Garbet.

\subsubsection{Características de las viviendas}

La vivienda es de mercado ordinario, de una o dos habitaciones, para una persona o dos como máximo -en caso de pareja o relación de amistad positiva-. Están equipadas con mobiliario, menaje, electrodomésticos y ropa del hogar, y distribuidas por la ciudad, no concentradas en un solo edificio.

El contrato de alquiler puede ir a nombre del gestor o del propio participante; en el caso que vaya a nombre del gestor (como a la práctica han sido todos los casos), se tendrá que analizar la idoneidad del cambio del titular a nombre del participante. El participante firma un Contrato Social con la empresa gestora, donde se recogen los compromisos básicos. Se le entrega un inventario del menaje de la vivienda, una copia del contrato de alquiler del gestor con el propietario, la normativa de la comunidad de vecinos (si existe) y un documento que explicita la aportación económica del participante, que será de un máximo del $20 \%$ de sus ingresos anuales.

Respecto a la búsqueda de los pisos, las entidades adjudicatarias debían aportar al programa pisos del mercado privado de alquiler (24 pisos -12 por lotedurante los primeros 7 meses, y el resto de viviendas, en los siguientes 5 meses). La búsqueda de los pisos adecuados para el programa se ha considerado una de las dificultades principales hasta la fecha.

\subsubsection{Equipo profesional}

El equipo de Primer la Llar es multidisciplinar y consta de un equipo social, integrado por educadores sociales, trabajadores sociales, integradores sociales y pares en calidad de expertos; y un equipo de salud mental, integrado en el Equip de Salut Mental per a persones Sense Sostre (ESMESS) ${ }^{6}$, formado por psiquiatras, enfermeros, y un profesional de acompañamiento en salud mental del Programa de Seguimiento Individualizado. La ratio de profesionales del campo social es de una por cada diez personas, más cinco horas a la semana (de media) por piso de integrador social o par. El par forma parte del equipo, es un profesional más, que ha sido formado y capacitado, y que en su trayectoria vital ha pasado por una situación de sinhogarismo.

El equipo social incluye tres perfiles diferentes: el de trabajador social o educador, para las funciones de atención educativa; el de técnico en integración social, para el soporte instrumental a la vida diaria; y el de par, que compaginaría funciones de soporte instrumental con funciones de observación y análisis, y funciones de referencia o motivación para los participantes. Cada uno de los dos equipos sociales del programa (uno en cada lote) se ha configurado con tres profesionales del campo social, dos integradores, y uno o dos pares. El equipo debe garantizar atención a las urgencias las 24 horas, 365 días.

\subsection{Metodología de intervención}

El programa Primer la Llar utiliza una metodología mixta entre la gestión intensiva de casos (ICM)7 y el tratamiento asertivo comunitario (ACT), sobre todo con perfiles de alta necesidad. Dentro del equipo Primer la Llar, el equipo social y de salud son equipos diferenciados, aunque comparten dinámicas y espacios de trabajo de forma regular, y estrechas coordinaciones. Se pretende establecer dinámicas que los aproximen al tratamiento asertivo comunitario, pero con una orientación concertada en el abordaje del caso.

Los profesionales del campo social actúan como referentes de los participantes y como soporte del proceso de acompañamiento, aunque cualquier miembro del equipo social o sanitario puede desarrollar esta función en caso de que se considere adecuado en el desarrollo del trabajo interdisciplinar en equipo. El acompañamiento socioeducativo se desarrolla en tres momentos: detección y acceso a la vivienda, adquisición de autonomía, y proceso de salida del programa.

\subsection{Evaluación}

Primer la Llar se ha planteado como un proyecto piloto con evaluación cualitativa y evaluación de efectividad del programa a través de un diseño experimental. Este tipo de planteamiento nos va a permitir obtener datos sobre los perfiles, la intensidad de la intervención o la coordinación de los distintos agentes, de acuerdo con las condiciones

\footnotetext{
${ }^{6}$ Equipo de Salud Mental para Personas Sin Hogar.

${ }^{7}$ Intensive case management.
} 
locales (atención sanitaria, servicios sociales, acceso a vivienda, trabajo interdisciplinar, marcos legales, cultura organizativa); nos va a facilitar, asimismo, la toma de decisiones en cuanto a cómo hemos de desarrollar el modelo Housing First en la ciudad y a cómo encaja dentro del modelo global de intervención con personas en situación de sinhogarismo.

La obtención de resultados finales está prevista, en el caso de la evaluación de impacto, para finales de 2018, y en el caso de la evaluación cualitativa, para finales de 2019.

\subsubsection{Evaluación de impacto}

La evaluación de impacto pretende valorar la efectividad del modelo Housing First respecto al modelo tradicional que se ofrece en la ciudad de Barcelona. La lleva a cabo la entidad externa experta en evaluaciones Ivàlua-Institut Català d'Avaluació de Polítiques Públiques, mediante un diseño experimental con grupo de comparación asignado aleatoriamente. Utilizando dos grupos diferenciados de personas -el grupo programa, que son las personas que se incorporan a las viviendas, y el grupo comparación, que son aquellas que se mantienen en el circuito tradicional-, se prevé comparar tres áreas de la vida de las personas: la salud y calidad de vida, el empoderamiento y recuperación de habilidades, así como la participación y soporte social.

Los cuestionarios empleados para la evaluación de impacto son instrumentos validados. Para la evaluación de la salud y calidad de vida, se ha utilizado el cuestionario SF-36; para evaluar el empoderamiento y recuperación de habilidades, los cuestionarios General Self-Efficacy Scale y Story; y para evaluar la participación y soporte social, el cuestionario de red social. Estos instrumentos recogerán las respuestas tanto de los participantes del grupo programa como de los participantes del grupo control en tres momentos distintos -la presentación de la candidatura, a los 18 meses y a los 30 meses-, para poder así medir la evolución y el impacto del programa, y compararlo con la alternativa tradicional de tratamiento.

Se validaron un total de 141 casos, y se procedió seguidamente al sorteo, que fue realizado por la entidad externa Ivàlua mediante una asignación aleatoria de los candidatos en tres grupos: grupo programa (50 personas), grupo comparación (70 personas) y grupo reserva (19 personas). Se comunicó el resultado a los candidatos a través de la entidad o servicio que los propuso, y empezó la incorporación a los pisos de los candidatos del grupo programa, lo cual se hizo gradualmente a lo largo de un año.

Asimismo, se evaluará también la implementación del programa: cómo se ha desarrollado en relación con las previsiones iniciales, qué factores imprevistos y dificultades prácticas han surgido, y cómo se han resuelto. Gracias a un sistema de monitorización, se podrá explorar los datos recogidos, que se complementarán con entrevistas en profundidad a los implicados, tanto profesionales como participantes.

\subsubsection{Evaluación cualitativa}

La evaluación cualitativa la está realizando la Universidad de Barcelona ${ }^{8}$, y se llevará a cabo desde dos vertientes diferenciadas y complementarias: un primer análisis sobre el impacto del programa en los participantes y un segundo sobre la intervención de los profesionales.

Respecto al impacto del programa en los participantes, se mostrarán los efectos que el acceso a una vivienda estable, combinado con un soporte intensivo a través de un equipo multidisciplinar, tiene en las personas con respecto a su recuperación y bienestar desde una perspectiva global. Se utilizará un método biográfico para conocer las experiencias de las personas que han llegado a una situación de sinhogarismo, a través de una muestra aleatoria: se realizaran entre 20 y 25 entrevistas en tres momentos diferentes del proceso (cuando la persona se incorpora, a los 18 meses y a los 3 años). En la implementación de programas Housing First, se ha identificado la soledad como una de las dificultades de residir en una vivienda de forma individual y las dificultades para la creación de nuevas relaciones sociales, por lo que se analizará en particular este aspecto. Los cambios en la socialización están asociados tanto a la autopercepción de la calidad de vida de los individuos como a la configuración del capital social necesario para afrontar situaciones vitales complicadas.

En cuanto a la intervención de los profesionales, se realizará una investigación-acción que facilite la transformación práctica de los equipos profesionales, según los principios del modelo Housing First. La metodología está basada en la teoría fundamentada (grounded theory), un método inductivo que concibe la práctica como un proceso complejo que se puede observar, sistematizar y acompañar para facilitar el cambio. Se utilizará la técnica del relato de práctica, que ya ha sido empleada en Canadá con objetivos similares y que permite que profesionales e investigadores desarrollen su capacidad de análisis. A los profesionales, les ayudará a comprender mejor el modelo a través de las situaciones concretas, les ayudará a ser coherentes entre el discurso y sus acciones reales, con las tensiones, dilemas éticos, límites y contradicciones que emergen de la práctica.

\footnotetext{
${ }^{8}$ Desde 2012, la Universidad de Barcelona está en estrecha colaboración con el Centre de Recherche de Montréal sur les inégalités, les discriminations et les pratiques alternatives de citoyenneté (CREMIS), que ha realizado la evaluación cualitativa del Proyecto Chez Soi/At Home en Montreal.
} 
A los investigadores, les permitirá identificar los aspectos críticos para la transformación de práctica y la definición de los instrumentos para acompañar al cambio. Se combinarán encuentros con todo el equipo con un trabajo más etnográfico, a través de observaciones y entrevistas a los equipos.

\subsubsection{Evaluación Outcomes Star}

Outcomes Star es un sistema de evaluación de origen inglés que está basado en la descripción de variables graduadas de manera sistematizada (de menor a mayor mejora o autonomía) y que, por tanto, permite evaluar la evolución de una situación con los mismos parámetros. El sistema dispone de instrumentos específicos ('estrellas') para estudiar diferentes ámbitos o colectivos, y en este caso, se utilizará el diseñado para el sinhogarismo, que evalúa diez dimensiones (como la motivación, las habilidades, la gestión económica, la salud física, la salud mental, el uso del tiempo, la gestión del alquiler o las transgresiones), en cada una de las cuales se examinan diez variables o situaciones. El Sistema de Evaluación Outcomes Star nos permitirá estandarizar el seguimiento de la evolución del participante (en concreto, su grado de autonomía) a lo largo del programa, e incorporar los datos resultantes al sistema informático municipal.

Esta evaluación permite no sólo evaluar de manera muy gráfica (estrella numérica) el proceso de evolución observado por los profesionales, sino también que los propios participantes puedan realizar una autoevaluación.

\subsubsection{Evaluación de fidelidad al modelo Housing First}

El modelo Housing First basado en la metodología Pathways to Housing incorporó una escala de autoevaluación (Pathways HF Fidelity SelfAssessment Scale), compuesta por 38 ítems agrupados en 5 áreas: proceso y estructura de la vivienda, vivienda y servicios, filosofía del servicio, oferta de servicios y estructura del equipo. Aunque cumplir al máximo los ítems definidos en el modelo norteamericano no ha sido objetivo principal del programa Primer la Llar, se realizó un análisis interno sobre su ajuste a la fidelidad descrita en Pathways to Housing.

Esta autovaloración de fidelidad de Primer la Llar a los principios centrales del modelo original de Pathway to Housing en los EE.UU. muestra un alto grado de fidelidad global ( $81 \%)$. La fidelidad más alta se encuentra en los campos de la filosofía del servicio ( $98 \%$ ) y en la estructura del programa (87\%); es menor en relación con la separación de alojamiento y servicios ( $83 \%$ ), y la variedad de servicios ( $76 \%$ ); y el grado más bajo de fidelidad se da en el campo de la elección del alojamiento y la estructura (57\%). No obstante, ésta es sólo una aproximación orientativa e interna.
En Europa, se han desarrollado varias experiencias basadas en el modelo Pathways to Housing norteamericano (Busch-Geertsema, 2013), pero también se han abierto nuevas miradas a modelos más flexibles, que incorporan la posibilidad de viviendas en el mismo edificio, incluso compartiendo servicios comunes, tal y como ha desarrollado $Y$ Foundation en Finlandia9.

\section{Primeras valoraciones y reflexiones}

A marzo de 2017, disponemos de datos de seguimiento del programa, de la evaluación realizada por Ivàlua referida al momento inicial, y a la evaluación cualitativa de seguimiento de la implementación. Por otro lado, la Universidad de Barcelona ha empezado a entrevistar a las 25 personas que forman parte del estudio de historias de vida y asimismo se ha empezado el trabajo metodológico para sustentar el cambio en el tipo de intervención.

\subsection{Perfil de los participantes}

Una vez realizadas las derivaciones de los usuarios al programa, se ha procedido al análisis de los perfiles de los 50 participantes:

- El $59 \%$ lleva entre 3 y 19 años durmiendo en la calle; el $14 \%$, entre 20 y 30 años; y sólo un $27 \%$, entre 1 y 2 años. En cuanto al uso de recursos de alojamiento, el $67 \%$ los ha utilizado entre 3 y 19 años; el $5 \%$, entre 20 a 25; y el $28 \%$, de 1 a 2 años.

- Respecto al estado de salud, un $48 \%$ presenta drogodependencia; un $24 \%$, enfermedad mental; y un $28 \%$, patología dual.

- En lo que se refiere a la edad de los participantes, la franja predominante es la que va entre los 41 y los 60 años, que concentra al $62,38 \%$ de las personas. La media de edad se sitúa en los 46,5 años. El participante más joven tiene 20 años, y el mayor, 79 .

- El $80 \%$ de los participantes son hombres, y el $20 \%$, mujeres.

- Por lo que respecta a la nacionalidad, los españoles suponen el $67 \%$. Entre los extranjeros, el $9 \%$ son de Marruecos; el $4 \%$, de Bulgaria; el $2 \%$, de Rumanía; y otro $2 \%$, de Argentina. El resto de nacionalidades son muy variadas.

\subsection{Datos de seguimiento}

De los primeros resultados del seguimiento técnico a lo largo de 2016, así como de los resultados obtenidos del análisis basal realizado por Ivàlua, destacamos los siguientes:

9 Y Foundation. Organización que pretende erradicar el sinhogarismo en Finlandia asegurando el acceso a la vivienda asequible, opera en 52 ciudades finlandesas y dispone de 6.675 apartamentos. 
- Las personas participantes (9 mujeres y 41 hombres) se mantenían en el programa, habiéndose producido una defunción.

- Cuarenta y dos de los cincuenta participantes disponían de ingresos y contribuían al pago del alquiler.

- A enero de 2016, sólo uno de los participantes había solicitado tener pareja en su piso. A diciembre de 2016, eran quince los participantes con pareja que compartían piso, aunque sólo cinco compartían gastos de alquiler.

- Dos participantes con enfermedad mental grave se cambiaron de vivienda por no encajar en la dinámica de la comunidad de vecinos.

- Durante 2016, se realizaron 154 visitas del equipo de salud mental (ESMESS). Se realizaron 27 ingresos hospitalarios, 3 ingresos psiquiátricos y 4 ingresos para desintoxicación. Además, hubo una llamada al 061.

- Durante 2016, se realizaron 1.005 visitas a domicilio y 491 fuera del domicilio, 603 atenciones telefónicas y 105 atenciones fuera del horario habitual (atenciones urgentes con desplazamiento del profesional).

En cuanto a la evaluación de impacto, y a la vista del primer análisis de Ivàlua que analiza la información basal relativa a los datos estudiados -calidad de vida relacionada con la salud, autoeficacia, recuperación personal y relaciones sociales-, todos los valores obtenidos son peores que los valores de referencia de la población española. En cuanto a la salud, las diferencias son importantes tanto en las dimensiones de salud en general como en la salud mental, y cabe destacar que las mujeres de la muestra están relativamente peor que los hombres.

\subsection{Valoraciones sobre la implementación}

Para complementar la información de los datos de las encuestas basales, Ivàlua ha realizado una evaluación cualitativa de seguimiento de la implantación (Sanz y Vílchez, 2017) en diciembre de 2016, en donde se recogen las primeras valoraciones de los usuarios y los profesionales del programa, a través de entrevistas. Los usuarios valoran sobre todo el hecho de vivir en una vivienda, destacando aspectos como la intimidad, la seguridad o la estabilidad. El personal técnico ya valora algunos avances puntuales en los ejes básicos de intervención, en general, en el campo de la salud, y en particular, en la adhesión voluntaria a los tratamientos.

- El inicio del reconocimiento de los problemas, la aceptación de visitas médicas, el seguimiento de las citas y las pautas médicas de los tratamientos, e incluso la adhesión voluntaria a tratamientos que impliquen ingresos temporales en instituciones para atender trastornos mentales severos 0 adicciones.
- Mejoras perceptibles en la apariencia física y el estado general de salud.

- Mejora del estado anímico y mayor disposición a expresar emociones e inquietudes, gracias al vínculo establecido entre el usuario y el profesional.

- Mejoras importantes en la autonomía personal en cuanto al cuidado personal y de la vivienda.

- Cambios en los ambientes sociales, alejamiento de relaciones vinculadas a espirales de deterioro y consumo de tóxicos y apertura progresiva a nuevas relaciones de proximidad.

De los primeros datos de la implementación presentados por Ivàlua (Sanz y Vílchez, 2017), destacaremos algunas aproximaciones referidas a la entrada en el programa, la vivienda y el acompañamiento socioeducativo.

\subsubsection{Entrada en el programa}

La implementación gradual del programa ha permitido un proceso muy cuidado de acogida y entrada de los participantes en las viviendas. El primer contacto con el usuario por parte del equipo Primer la Llar se hace en coordinación con el referente derivante. Se presenta el programa en el centro derivante 0 en la vivienda, a elección del usuario. Después, se le muestran un mínimo de dos viviendas, para que pueda escoger. Se entregan las llaves, y el participante decide si se queda de inmediato en el piso o necesita unos días antes de mudarse. Una vez el participante se incorpora a la vivienda, se procede a la firma del contrato social, recordando las condiciones de uso de la vivienda y los mínimos compromisos que ya se aceptaron, y se firma el anexo económico, donde se regula la aportación económica al alquiler. A partir de entonces, se inician las visitas, en principio semanales, como mínimo, pero que se adaptarán a las necesidades y requerimientos del participante, $y$ que podrán ser en el mismo domicilio o en otro lugar acordado.

El orden de entrada de los casos se hizo de manera consensuada, priorizando en principio aquellos casos de una mayor vulnerabilidad y aquellos que estaban en situación de calle. Sin embargo, aspectos más prácticos, como las características de los pisos y las preferencias de zona de los usuarios, hacían unos pisos más adecuados para unos usuarios que para otros e influyeron también en la priorización de las entradas. En cuanto a la elección entre dos viviendas, las personas han optado en términos generales por quedarse con el primer piso que se les enseñaba.

\subsubsection{Sobre la vivienda}

Los dos gestores han utilizado recursos estructurales de sus propias organizaciones o de entidades vinculadas a ellas para la búsqueda de los pisos, que ha resultado muy complicada. Los factores que 
explican esta dificultad no han sido únicamente el elevado precio del alquiler en Barcelona, que continúa en alza, sino también la desconfianza de los administradores de fincas hacia los proyectos sociales y, en particular, hacia los destinados a personas sin hogar, así como la importante demanda de vivienda asequible en Barcelona.

En las estrategias argumentales utilizadas por los gestores con las agencias inmobiliarias y propietarios, se destacaban las siguientes ventajas: garantía de pago y del buen estado del piso, un equipo de soporte comprometido a intervenir ante cualquier problema de convivencia, y el valor asociado de colaborar en un proyecto innovador de soporte a las personas sin hogar. Mantener una buena relación y un alto grado de respuesta a las preocupaciones de los propietarios, agencias de pisos y comunidades de vecinos ha sido clave para implementar el programa.

Se han llevado a cabo mediaciones con las comunidades de vecinos y con la propiedad, se ha demostrado predisposición a la comunicación y la colaboración (facilitándose en algunas ocasiones los teléfonos de los miembros del equipo) y siempre se han atendido las situaciones límite de convivencia, trasladando rápidamente a la persona a otro piso diferente (en dos casos). Se ha conseguido reconducir los conflictos y mantener la confianza de la comunidad, de forma que hasta el momento no se ha rescindido ningún contrato de alquiler.

\subsubsection{Sobre el acompañamiento socioeducativo}

En la puesta en marcha del servicio, se han establecido tres momentos: la detección y acceso a la vivienda por parte de las personas seleccionadas, la adquisición de autonomía y el proceso de salida del programa. Actualmente, el programa ha superado la primera fase de detección y acceso a la vivienda de las personas, y en todos los casos, se ha pasado a la fase de desarrollo de autonomía, aunque se observan diferencias al respecto: algunos participantes se sitúan en una fase muy inicial de reconocimiento de situación, mientras que otros han hecho un proceso más rápido de vinculación y presentan mayor autonomía. La fase de salida del programa no se plantea en ningún caso, ni se prevé antes de finalizar la prueba piloto (diciembre 2018).

Cuando las personas padecen procesos agudos de sus problemas de salud mental o adicciones, que pueden llegar a tensar el marco de las condiciones de acceso -en especial, por poner en riesgo la buena convivencia vecinal, la propia integridad o la habitabilidad del piso-, la práctica del acompañamiento se ha ajustado con un incremento de soporte y acompañamiento. Hasta el momento, no ha sido necesario ningún ingreso involuntario.

Cabe destacar también el hecho de que las actividades realizadas son de carácter individual, mientras que las grupales propuestas no han tenido demasiado interés, pues en algunos casos se ha constatado cierto rechazo a verse vinculados con personas que se encuentran en la misma situación.

\section{Fases de la intervención}

En la intervención centrada en la persona, hemos diferenciado tres fases: explosión de expectativas al entrar en el piso, aterrizaje y toma de conciencia de las dificultades de adaptación a la vida en un piso, y afrontamiento de las dificultades. Las dificultades más importantes en la adaptación a la vivienda estarían relacionadas con el espacio de vida habitual y la adquisición de hábitos básicos, como la adaptación a las condiciones del descanso nocturno, la dificultad para cocinar, la limpieza de la vivienda o los hábitos de higiene personal.

Sin embargo, la dificultad que más destacan tanto técnicos como participantes es la soledad: la pérdida de relaciones a lo largo del proceso de sinhogarismo, así como la posible ruptura de relaciones desarrolladas en la vida en la calle. Algunas personas en este proceso de adaptación continúan manteniendo algunas costumbres o vínculos de su etapa de sinhogarismo: quieren ir al comedor social, piden en la calle, o acuden a lugares donde les pueden facilitar ropa y calzado, por ejemplo.

El programa está todavía en una fase inicial en la que predominan las actividades básicas de soporte y de creación de vínculo. En cambio, las relativas a la recuperación o formación de nuevas relaciones sociales o la activación laboral están menos presentes.

\section{Equipos, pares y salud mental}

Los equipos celebran normalmente dos reuniones de coordinación a la semana para tratar aspectos organizativos (entradas, visitas, acompañamientos, incidencias), y el resto de la semana, las actividades se desarrollan básicamente en los pisos, aunque el contacto telefónico es constante. En cualquier caso, hay cultura de equipo y cohesión, así como absoluta flexibilidad y capacidad de reacción ante cualquier imprevisto. Se hacen también reuniones periódicas de reflexión sobre el modelo y el posicionamiento personal ante los casos que se tratan.

Respecto al perfil competencial, se han valorado como fundamentales la empatía, la capacidad de mediación y resolución de conflictos, y la experiencia en intervención social en el campo de las personas sin hogar, la drogadicción o la salud mental. Uno de los gestores ha apostado más por personas con experiencia en servicios convencionales para personas sin hogar, mientras que en el otro gestor dicha experiencia no ha estado tan presente, o hay al menos una mayor mezcla de perfiles. En un programa que rompe con la metodología tradicional, la 
experiencia es un aspecto ambivalente: por un lado, permite una capacidad de observación y análisis más refinada y una mayor seguridad en la respuesta; en cambio, una persona sin experiencia en el modelo tradicional puede incorporar la nueva manera de intervenir de una forma más natural y rápida, aunque probablemente menos consciente y analítica.

Sobre la figura del par, se ha valorado que el encaje, función y rol dentro del equipo no está todavía bien definido. Se considera que su valor diferencial sería observar y expresar consideraciones respecto a los casos, y en menor medida, la referencia o motivación para los participantes.

Respecto a la atención sanitaria, se procura el vínculo con los centros de atención primaria del barrio para la atención general a la salud, mientras que la atención a la salud mental se ofrece a través del ESMESS en una parte significativa de los casos. Si es necesario, se plantean visitas coordinadas y conjuntas del equipo social y del de salud mental. La coordinación con el ESMESS es estrecha tanto para realizar valoraciones conjuntas como para orientar el abordaje de alguna situación. Cuando la persona ya era atendida en un centro especializado de salud mental, se ha mantenido esa referencia, activándose la coordinación con dicho centro. En los casos de adicciones, el ESMESS actúa coordinado con los centros de atención y seguimiento de drogodependencias.

\section{Equipo municipal y técnico}

El programa Primer la Llar se encuentra en fase de construcción, y por tanto, el trabajo conjunto entre el Ayuntamiento y los gestores del servicio es clave. Aunque los parámetros de definición estaban descritos y fundamentados, su puesta en marcha permite ir ajustando el funcionamiento a partir de la misma práctica. A través de una estrecha coordinación, se han ido consensuando los diferentes aspectos que conforman la implementación del modelo: el proceso de entrada en la vivienda, el ajuste de las funciones de los diferentes profesionales, la definición sobre la práctica de cómo debe ser la intervención y la creación del vínculo, las sesiones de los casos, la evolución de los participantes y cómo medirla, entre otras cuestiones.

El Ayuntamiento se reúne periódicamente (como mínimo, un vez al mes) con los dos gestores, y se van alternando las sesiones de casos con las sesiones organizativas; en función del contenido de la reunión, también se cuenta con el ESMESS. Las reuniones fueron especialmente intensivas en las fases iniciales del proceso, realizándose incluso sesiones monográficas sobre aspectos concretos: el programa es un espacio de discusión abierto y horizontal donde las decisiones se toman por consenso. Al margen de las reuniones, los contactos son constantes entre el Ayuntamiento y los gestores, vía correo electrónico o teléfono.
Durante el proceso de implementación, hay aspectos de funcionamiento y metodología que se han de ajustar con la práctica, y entre los que se han ido poniendo encima de la mesa están la cuestión de las parejas, el diseño del contrato social o las ayudas económicas. Asimismo, se juzga muy positivamente la implicación y motivación de los profesionales y el continuo intercambio de información que enriquece la intervención de los dos gestores.

Por otro lado, hay un segundo espacio para trabajar específicamente con el ESMESS los casos concretos que están bajo su supervisión o tratamiento, que son los que presentan una mayor complejidad en lo que se refiere a salud mental o drogodependencias.

El trabajo es intenso para llegar a consensos donde inicialmente puede haber disparidad de opiniones entre el equipo social y el de salud mental, pero es necesario ir forjando la perspectiva de un único equipo de trabajo entre los dos.

Aparte de las reuniones de seguimiento, mensualmente se recoge un extenso conjunto de indicadores informatizados sobre la actividad del servicio, la situación de los pisos y el estado de las personas beneficiarias en relación con los ejes principales de la intervención. A partir de octubre, se iniciará la evaluación mediante el sistema Outcomes Star.

\section{Relación del equipo con otros servicios del barrio}

No se hace una presentación previa del programa a otros servicios del barrio, a no ser que el usuario lo pida expresamente, pues en todo momento se busca el protagonismo y empoderamiento de la persona. No es fácil ejercer el rol de referente y de acompañamiento, especialmente en las relaciones con los demás servicios sociales, puesto que, por un lado, el acompañamiento puede ser un recurso del usuario para mejorar el trato en el servicio, o facilitar el contacto entre profesionales, pero a la vez limita la confianza y el crecimiento de sus capacidades. La integración y trabajo conjunto entre los profesionales del programa Primer la Llar y los profesionales del barrio cuando la persona usuaria del programa así lo ha demandado no ha presentado dificultades de encaje en el modelo Housing First hasta el momento.

\section{Metodología de intervención}

La composición y número de profesionales se valora hasta el momento adecuada teniendo en cuenta que no todos los casos requieren un abordaje mediante tratamiento asertivo comunitario (ACT) y que se están combinando con intervenciones no tan intensas, situación que permite mantener un equilibrio en la dotación de profesionales y la intensidad de la intervención.

Los profesionales mantienen una elevada implicación en todo el proceso de implementación y valoran la 
experiencia como un gran proceso de aprendizaje y de abordaje de la atención a las personas sin hogar. Tal y como expresa uno de los profesionales entrevistados:

Es un trabajo de aprender y también de desaprender lo que habías hecho [...]. Es muy diferente estar en un espacio reglamentado que estar en casa de la persona, te permite una forma de relación distinta [...]. Lo que has de desaprender es que tú no tienes la razón y tú no sabes... Desaprender la inercia profesional a tener la razón, a guiar, a ser directiva... En una relación de igual a igual, la legitimidad [de] que la persona escuche lo que yo quiero ofrecerle... eso me lo tengo que ganar, no es porque yo sea trabajadora social... (Sanz y Vílchez, 2017).

Se han planteado cambios de nomenclatura, como 'participante', en lugar de 'usuario', 'plan de soporte o acompañamiento' en lugar de 'plan de trabajo', o 'equipo de salud' mental en lugar de 'equipo psiquiátrico’.

\section{Sobre los participantes}

Hasta el momento, la impresión global de los entrevistados en el proceso de implementación es muy positiva. Algunos participantes manifiestan abiertamente los aspectos favorables del modelo: estar en su propia casa, seguridad, recuperación de relaciones sociales, disminuir el consumo de alcohol, aceptar tratamiento sanitario. Otros participantes, aun presentando graves dificultades de salud, o manteniendo el uso y contacto con los servicios vinculados a la atención de personas sin hogar (comedores sociales, servicios de higiene), expresan su voluntad de continuar en la vivienda y de mantener el vínculo con los profesionales:

Yo lo que quiero es estar aquí, portarme bien con estas personas que se portan doblemente bien conmigo... Lo que quiero es buscar trabajo..., he sido encargado... En mi vida laboral, consta que he cotizado muchos años... Yo aquí (en Barcelona) no valgo para nada (Sanz y Vílchez, 2017).

\subsection{Reflexiones finales}

Después de un año y medio de la puesta en marcha del programa Primer la Llar y sin disponer aún de datos definitivos de las evaluaciones iniciadas, podemos posicionarnos de manera positiva respecto del funcionamiento, tanto desde la perspectiva de los participantes, de los profesionales implicados como del encaje del proyecto Housing First en el contexto de la ciudad de Barcelona.

Todos los participantes se mantienen en su piso, ninguno quiere volver a la situación anterior. Tras un año de permanencia en la vivienda, el $30 \%$ ha iniciado relaciones sentimentales, ha disminuido consumos 0 entrado en tratamiento; algunos mantienen vínculos con su entorno anterior y otros los han roto, siendo plenamente conscientes de la opción que han tomado; las viviendas se mantienen en condiciones tanto con el entorno como en su interior; y la relación con los profesionales es estrecha.

En cuanto a los profesionales, están en actitud constante de observación activa y predispuestos al cambio, contrastando dudas y compartiendo dificultades. Los casos más complicados, que exigen mayor intensidad de intervención de la prevista, se pueden equilibrar con las situaciones que requieren una menor atención, puesto que la recuperación de la persona ha sido muy rápida. La relación entre el equipo de salud y el equipo social es cada vez más estrecha.

A la espera del análisis comparativo de evolución de resultados, así como del análisis del cambio metodológico, resaltamos algunos elementos dignos de considerar con perspectiva de futuro:

- En el desarrollo del programa, destacamos las dificultades para conseguir viviendas, que han complicado la puesta en marcha del programa, puesto que han requerido un sobresfuerzo a los gestores para vencer las resistencias de los propietarios y administradores, y poder contratar pisos a precios asequibles en un mercado como el de Barcelona, con constantes subidas del alquiler. En este sentido, replicar la experiencia o escalarla no será fácil si el acceso a la vivienda no se facilita de alguna manera. La vivienda es una necesidad básica tanto para quienes se encuentran en situación de sinhogarismo como para quienes están en exclusión residencial o cuentan con un alojamiento precario, inseguro o inadecuado.

- El nivel de ingresos de las prestaciones a las que pueden acceder las personas usuarias es insuficiente para poder garantizar la plena autonomía económica en la ciudad. Sería necesario un acompañamiento de políticas de vivienda, concretamente de ayudas al alquiler que pudieran facilitar que aquellas personas que consigan una autonomía suficiente, pero dispongan de ingresos bajos y una difícil integración al mercado laboral, tengan posibilidades reales de mantenerse en los pisos.

- Una estrecha coordinación, la constante discusión e intercambio de información, así como la búsqueda de consensos a la hora de encontrar la mejor solución para cada caso es la clave para el seguimiento de una metodología que debe respetar las decisiones de los usuarios, ofreciendo el acompañamiento más adecuado en cada situación. La necesidad de redefinir la profesión y de ajustar la formación y los sistemas metodológicos de intervención son ejes básicos para asegurar una actuación con fundamento teórico y práctico.

- Otros aspectos que pueden condicionar el enfoque programa - unos que ya han empezado 
a aparecer en la casuística y otros que se han planteado como hipótesis- son la formación de parejas, los posibles embarazos, las situaciones de violencia machista o los reagrupamientos familiares. También los ingresos hospitalarios largos o los ingresos en prisión. Preocupan en especial aquellos casos con trastorno mental grave y sin voluntad de tratamiento.

- A partir de la evaluación de los resultados que se obtengan con la implantación del programa y su comparación con la alternativa tradicional, podremos establecer unos indicadores que permitan orientar la continuidad del modelo. La cuestión será si el modelo Housing First debe únicamente destinarse a los colectivos más excluidos y en situación más crónica, o debe ampliarse a otros, como jóvenes o familias (Housing Led).

Tal como apunta Volker Busch-Geertsema en la revisión por pares (peer review) que HABITACTFeantsa realizó el 3 y 4 de noviembre de 2016 en la ciudad de Barcelona:

A pesar de que el sistema de soporte para las personas sin hogar en Barcelona es uno de los más destacados del Estado Español y muy importante en el ámbito europeo, presenta sin embargo algunas dificultades, como el resto de sistemas de escala en todo el mundo:

- El bloqueo en el extremo superior del sistema a la hora de encontrar alojamiento permanente para aquellas personas que han progresado en la escala.
- El efecto de puerta giratoria, puesto que hay personas que se encuentran nuevamente en los niveles más bajos de la escala.

- Finalmente, aquellas personas en situación de sinhogarismo crónico que han recibido un soporte inadecuado a sus necesidades no han conseguido avanzar y continúan marginadas.

La presión asistencial en Barcelona (con 1.907 personas durmiendo en recursos especializados para las personas sin hogar, y 941 personas durmiendo en la calle contabilizadas en 2016) [XAPSLL, 2016], junto con la escasez de vivienda social y de vivienda asequible, comporta que, en la ciudad, el modelo Housing First tenga que coexistir con el modelo tradicional. El encaje del modelo Housing First con el modelo de escala con el que ha de coexistir es complicado, pues son enfoques metodológicos distintos. Housing First tiene como ejes fundamentales tanto el derecho a la vivienda como el derecho a la autodeterminación del usuario, como sujeto con plenos derechos. A pesar de que en el modelo en escala el papel del usuario en el propio proceso cada vez está más presente, los tiempos de las estancias máximas en los equipamientos se están flexibilizando y se trabaja para que los espacios individuales tengan cada vez un mayor peso en la cartera de recursos de atención de las personas que están en situación de sinhogarismo, tenemos que trabajar para superar la perspectiva tradicional de la atención a este colectivo y orientar la intervención de los servicios sociales hacia la reconstrucción de un hogar donde puedan desarrollar sus trayectorias vitales. 
AJUNTAMENT DE BARCELONA (2015): Pla de Lluita contra el Sensellarisme de Barcelona. 2016-2020, Barcelona, Ajuntament de Barcelona.

BUSCH-GEERTSEMA, V. (2016): Peer Review on Homelessness Policies in Barcelona, Spain, Habitact.

- (2013): Housing First Europe. Final Report, Bremen; Bruselas, Comisión Europea [rhttp:// www.giss-ev.de//files/giss/upload/Pdf/ FinalReportHousingFirstEurope.pdf $\rangle]$.

FORTEA, C. (2005): Programa Municipal d'Atenció a Persones sense sostre, Barcelona, Ajuntament de Barcelona.

GOBIERNO DE ESPAÑA (2015): Estrategia Nacional Integral para Personas sin Hogar 2015-2020, Madrid, Gobierno de España.

PLEACE, N. (2012): Le logement d'abord, París, DIHAL; European Federation of National Organisations Working with the Homeless.

SALES, A. (2015): "How many homeless people live in Spain? Incomplete sources and impossible predictions", European Journal of Homelessness, vol 9, no 2 [khttp://www. feantsaresearch.org/download/salesejh22015article96043744236334400007.pdf)]

SANZ, J.; y VÍLCHEZ, D. (2017): Avaluació del programa 'Primer la Llar' de l'Ajuntament de Barcelona. Avaluació qualitativa de la implementació, Barcelona, Ivàlua.

TSEMBERIS, S. (2010a): "Housing First: ending homelessness, promoting recovery and reducing costs", en GOULD ELLEN, I; y O'FLAHERTY, B. (eds.), How to House the Homeless, Nueva York, Russell Sage Foundation.

- (2010b): Housing First. The Pathways Model to End Homelessness for People with Mental Illness and Addiction, Center City, Hazelden.

TSEMBERIS, S.; y EISENBERG, R. F. (2000): Pathways to Housing: Supported Housing for Streetdwelling Homeless Individuals with Psychiatric Disabilities, Washington D.C., Psychiatric Services.

XAPSLL (2016): “El recuento de 2016”, Barcelona, Xarxa d’Atenció a Persones Sense Llar [<http:// sensellarisme.cat/es/el-recuento-de-2016/১]. 


\section{Bibliografia complementaria}

(2016): Barcelona Societat. Revista de Coneixement i Anàlisi Social, $\mathrm{n}^{0} \mathbf{2} 2$.

AJUNTAMENT DE BARCELONA (2017): Primer la Llar. Episodi 1 [vídeo] ['https://youtu.be/21t57JbDsjo〉].

- (2017): Primer la Llar. Episodi 2 [vídeo] [〈https://youtu. be/_xiPzexRybs〉].

ASUNTO ENSIN (2011): Avaimet (Housing First: The Keys) [vídeo] [/https://youtu.be/cQPdXTc5Qel>].

AUBRY, T. et al. (2015): “One-year outcomes of a randomized controlled trial of housing first with ACT in five Canadian cities", Psychiatric Services, vol. 66, no 5 ['https://doi.org/10.1176/appi. ps.201400167>].

BERNAD, R.; YUNCAL, R.; y PANADERO, S. (2016): "Introducing the Housing First model in Spain: First results of the Habitat Programme", European Journal of Homelessness, vol. 10, $\mathrm{n}$ 1 , págs. 53-82.

BUSCH-GEERTSEMA, V. et al. (2010): Homelessness and Homeless Policies in Europe: Lessons from Research. A Report for the European Consensus Conference on Homelessness, Bruselas, European Federation of National Organisations Working with the Homeless.

CABRERA, P., et al. (2008): Qui dorm al carrer? Una investigació social i ciutadana sobre les persones sense sostre, Barcelona, Fundació Caixa Catalunya.

COMISIÓN EUROPEA (2013): Paquete de inversion social. Documento de trabajo de los servicios de la Comisión 'Abordar el sinhogarimo en la Unión Europea', Bruselas, Comisión Europea, 20-2-13, SWD(2013) 42 final.

ESTECAHANDY, P. (2015): “The French experimentation 'Un chez soi d'abord'. From social experiment to public policy" [presentación], Conferencia Internacional Hábitat 'New experimentations on evidence based Housing First', octubre.

FARHA, L. (2015): Informe de la Relatora Especial sobre una vivienda adecuada como elemento integrante del derecho a un nivel de vida adecuado y sobre el derecho de no discriminación a este respecto, Naciones Unidas, Asamblea General.

FEANTSA (2013): On the Way Home? FEANTSA Monitoring Report on Homelessness and Homeless Policies in Europe, Bruselas, European Federation of National Organisations Working with the Homeless.

FERNÀNDEZ EVANGELISTA, G. (2015): “El acceso a la vivienda social de las personas sin hogar. Estudio de casos: Alemania, España, Finlandia y Reino Unido" [tesis doctoral] Universidad Autónoma de Barcelona (Instituto de Gobierno y Políticas Públicas) [<http://www.tesisenred.net/ bitstream/handle/10803/368566/gfe1de2. pdf? sequence $=1$ ) $]$.

GAETZ, S.; SCOTT, F.; y GULLIVER, T. (eds.) (2013): Housing First in Canada: Supporting Communities to End Homelessness, Toronto, Canada Homelessness Research Network.

HOMELESS HUB, THE (s/d): Housing First: Definition [vídeo] [«https://vimeo.com/81543911)].

HURTUBISE, R. (2015): El Model Housing First en l'Abordatge del Sensellarisme [vídeo] [<https://www. youtube.com/watch?v=aceE47X_Ano〉].

MENTAL HEALTH COMMISSION OF CANADA (2014): Canadian Housing First Toolkit, Ottawa, Mental Health Commission of Canada.

- (2014): Rapport final du site de Montréal, Ottawa, Mental Health Commission of Canada. 
ORNELAS, J. (2013): Housing First Europe: Local Evaluation Report Lisbon, Bruselas, Comisión Europea [<http://www.giss-ev.de/files/giss/upload/ PDF\%20HFE/Lisbon_HFE_Local_Evaluation. $p d f>$.

PLEACE, N. (2016): Housing First Guide. Europe, European Federation of National Organisations Working with the Homeless [<http://housingfirstguide. eu/website/wp-content/uploads/2016/06/ HFG_full_Digital.pdf)].

SALES, A. (2013): Diagnosi 2013: les persones sense llar a la ciutat de Barcelona i l'evolució dels recursos de la Xarxa d'Atenció a les Persones Sense Llar, Barcelona, Xarxa d'Atenció a les Persones Sense Llar de Barcelona.

SALES, A.; URIBE, J.; y MARCO I. (2015): Diagnosi 2015: la situació del sensellarisme a Barcelona. Evolució i polítiques d'intervenció, Barcelona, Xarxa d'Atenció a les Persones Sense Llar de Barcelona.

TSEMBERIS, S. (2012): Ending Homelessness and Transforming Lives [vídeo] [<https://www. youtube.com/watch?v=HsFHV-McdPo>].

TULL, T. (2013): “Partnering for Change” [presentación] [<https://www.slideshare.net/UpSocial/ sicmonoparentals-tanya-tull-presentspartnering-for-change-eng>].

UNIÓN EUROPEA (2014): "Estrategia de la UE para las personas sin hogar. Resolución del Parlamento Europeo, de 16 de enero de 2014, sobre una estrategia de la UE para las personas sin hogar (2013/2994(RSP))", Diario Oficial de la Unión Europea, C 482, 23-12-16, págs. 141-144 [〈http://eur-lex.europa.eu/legal-content/ES/ TXT/?qid=1510853998161\&uri=CELEX:52014 IP0043'].

URIBE VILARRODONA, J. (2015): De la calle al hogar. Housing First como modelo de intervención y su aplicación en Barcelona, Esplugues de Llobregat, Ediciones San Juan de Dios.

VANDENBROUCKE, F. et al. (2011): European Consensus Conference on Homelessness: Policy Recommendations of the Jury, Bruselas, European Federation of National Organisations Working with the Homeless [<http://www. feantsa.org/download/2011_02_16_ final_consensus_conference_jury_ recommendations_en-22191246727835177211. pdf?force $=$ true . 